\title{
Estudo in vitro da influência do formato e do tratamento de superfície de implantes odontológicos no torque de inserção, resistência ao arrancamento e frequência de ressonância
}

\author{
In vitro study of the influence of the shape and surface treatment of dental implants in \\ insertion torque, pullout resistance and resonance frequency
}

\author{
Nathalia FERRAZ OLISCOVICZ ${ }^{\mathrm{a}}$, Mariana LIMA DA COSTA VALENTE, \\ Elcio MARCANTONIO JUNIOR ${ }^{\mathrm{b}}$, Antonio CARLOS SHIMANO ${ }^{c}$, Andrea CANDIDO DOS REIS ${ }^{\mathrm{a}}$
}

\begin{abstract}
${ }^{a}$ Faculdade de Odontologia, USP - Universidade de São Paulo, São Paulo, SP, Brasil ${ }^{b}$ Faculdade de Odontologia, UNESP - Univ Estadual Paulista, Araraquara, SP, Brasil 'Faculdade de Medicina, USP - Universidade de São Paulo, Ribeirão Preto, SP, Brasil
\end{abstract}

\begin{abstract}
Resumo
Objetivo: A proposta do estudo foi avaliar a influência do formato e do tratamento de superfície na estabilidade primária de implantes odontológicos, inseridos em diferentes substratos, utilizando-se associação de métodos, como torque de inserção, resistência ao arrancamento e frequência de ressonância. Material e método: Foram utilizados 32 implantes da marca Conexão ${ }^{\circledast}$ (Conexão Sistemas de Prótese Ltda, Arujá, São Paulo, Brasil), sendo: oito cilíndricos com tratamento Porous (CA), oito cilíndricos usinados (MS), oito cilíndricos tratamento duplo Porous (MP) e oito cônicos sem tratamento (CC). Os substratos utilizados para inserção foram: costela de porco; poliuretana Synbone ${ }^{\oplus}$; poliuretana $\operatorname{Nacional}^{\circledast}(15,20,40 \mathrm{PCF})$, e madeira. O torque de inserção (TI) foi quantificado utilizando-se um torquímetro digital Kratos $^{\circledR}$; a força de arrancamento (RA) foi aferida por meio de tração axial, realizada em uma Máquina Universal de Ensaios (Emic ${ }^{\circledast}$ DL-10000), e utilizou-se também análise por meio de frequência de ressonância (RF). Para obtenção dos resultados estatísticos, utilizou-se análise de variância e teste de Tukey (significância de 5\%). Resultado: Ao analisar o torque de inserção, verificou-se que os implantes com tratamento de superfície não foram diferentes estatisticamente dos usinados, assim como os implantes cilíndricos não tiveram diferença dos cônicos em todos os substratos ( $p>0,05)$, com exceção da poliuretana Synbone ${ }^{\oplus}$. Em relação à resistência ao arrancamento, os implantes tratados e usinados, assim como cônicos e cilíndricos, não tiveram diferença estatística ( $\mathrm{p}>0,05)$; a análise de frequência de ressonância mostrou que não houve diferença entre os implantes ( $p>0,05)$, com exceção da poliuretana Nacional ${ }^{\circledR}(20 \mathrm{PCF})$. Conclusão: Os formatos e o tratamento de superfície estudados não demonstraram valores significantes quando foram comparados os implantes entre si e, considerando os substratos avaliados, não houve diferença estatística entre os diferentes tipos de implantes.
\end{abstract}

Descritores: Implantes dentários; torque; substitutos ósseos; osseointegração.

\begin{abstract}
Objective: The purpose of the study was to evaluate the influence of the shape and surface treatment on the primary stability of dental implants inserted in different substrates through association methods such as insertion torque, pullout strength and resonance frequency. Material and method: 32 implants were used with 8 cylindrical treatment Porous (CA), 8 machined cylindrical (MS), 8 cylinder dual treatment Porous (MP) and 8 tapered untreated (CC). The substrates used for inclusion were: pork rib; ${ }^{\circledR}$ Synbone polyurethane, polyurethane National ${ }^{\circledR}(15,20,40$ PCF) and wood. The insertion torque (TI) was quantified using a digital torque $\mathrm{Kratos}^{\circledast}$ wrench, the pullout strength (RA) was measured by means of axial traction performed in an Emic DL- $10000^{\circledR}$ and analysis was also used by the resonance frequency (RF). To obtain the statistical results, we used analysis of variance and Tukey's test (5\% significance). Result: To analyze the insertion torque, it was found that implants with surface treatment were not statistically different from machined as well as the cylindrical implants did not differ from tapered on all substrates ( $p>0.05)$, except ${ }^{\oplus}$ Synbone of polyurethane; in relation to RA, treated and machined implants as well as tapered and cylindrical, showed no statistical difference ( $p>0.05)$; FR analysis showed no difference between implants $(\mathrm{p}>0,05)$, with the exception of National ${ }^{\circledR}$ polyurethane (20 PCF). Conclusion: formats and surface treatment studied showed no significant values when compared implants together and considering the tested substrates showed no statistical difference between the different types of implants.
\end{abstract}

Descriptors: Dental implants; torque; bone substitutes; osseointegration. 


\section{INTRODUÇÃO}

A eficácia do tratamento reabilitador com implantes odontológicos aumentou significativamente sua aplicação na prática clínica ${ }^{1-3}$. Mas, para o sucesso dessa terapia, é necessário que ocorra a estabilidade primária do implante ${ }^{2,4,5}$, a qual impede a micromovimentação do parafuso após a completa inserção do mesmo no leito ósseo ${ }^{6}$, facilitando a cicatrização natural e a formação óssea efetiva, e melhorando a osseointegração ${ }^{7}$.

Para que a estabilidade primária aconteça, é preciso levar em consideração diversos fatores que interferem diretamente nessa condição, como a qualidade e a quantidade óssea no sítio cirúrgico, a técnica cirúrgica empregada, o formato e o tratamento de superfície, devendo-se dar uma atenção especial para os dois últimos fatores ${ }^{6,89}$.

O formato do implante está relacionado a dois aspectos fundamentais: a macroestrutura, que pode diferir quanto a tamanho, desenho, número e passo de roscas, e tipo de conexão protética, fatores estes explorados superficialmente na literatura ${ }^{10,11}$, e a microestrutura, caracterizada pelos tratamentos de superfície ${ }^{12}$. O formato dos implantes exerce grande influência na estabilidade primária, visto que este deve permitir a ligação direta entre o tecido ósseo e a superfície do mesmo, sem a interposição de tecido fibroso, garantindo uma distribuição uniforme das cargas mastigatórias quando estas são transmitidas para o tecido peri-implantar ${ }^{5,13,14}$.

Diferentes tipos de tratamentos de superfície são descritos na literatura, como o ataque ácido, o jateamento de partículas e a anodização da superfície; no entanto, todos estes têm como objetivo principal o aumento da rugosidade superficial do titânio, o que leva a uma melhor interação osso/implante, favorecendo a formação óssea por meio do aumento da atividade osteocondutora e osteogênica das células ${ }^{15,16}$.

Tendo em vista a influência dos diferentes fatores que interferem na estabilidade primária dos implantes e a sua importância para determinar o tipo de tratamento protético a ser realizado - imediato, mediato ou tardio -, se faz necessária uma análise qualitativa e quantitativa da estabilidade, para se obter uma maior previsibilidade da osseointegração.

Alguns métodos bastante utilizados para aferir a estabilidade primária são a análise de frequência de ressonância (FR), o torque de inserção (TI) e os ensaios mecânicos, como o de arrancamento (RA). O torque de inserção e a frequência de ressonância mensuram a estabilidade primária por meio de um valor numérico e podem ser usados clinicamente ${ }^{17-20}$; já os ensaios mecânicos, como a resistência ao arrancamento ${ }^{21}$, avaliam in vitro a estabilidade primária de acordo com a técnica cirúrgica, $\mathrm{o}$ formato do implante e a densidade do osso ${ }^{22,23}$.

A variabilidade na densidade apresentada pelos ossos humanos naturais ${ }^{24}$ dificulta sua utilização em estudos laboratoriais. Assim, para reproduzir as diferentes densidades ósseas e tornar o estudo mais fiel à realidade clínica, foi utilizada, no presente estudo, a costela suína como substrato, por esta ter o tecido ósseo com maior similaridade com o tecido ósseo humano. Além desse substrato, para validar uma metodologia ${ }^{9}$, foram utilizados a madeira e os ossos sintéticos de poliuretano.
Para controlar o formato e o tratamento de superfície, que são os fatores relacionados ao implante mais relevantes para a osseointegração, a proposta do presente estudo foi avaliar a influência dessas duas variáveis na estabilidade primária de implantes inseridos em diferentes substratos, por meio da associação de técnicas de torque de inserção, frequência de ressonância e resistência ao arrancamento ${ }^{6,25}$.

\section{MATERIAL E MÉTODO}

\section{Substratos para Instalação dos Implantes}

A instalação dos implantes e a realização das análises foram feitas em diferentes tipos de corpos de prova: osso de costela suína, madeira, osso artificial de poliuretana $\mathrm{Nacional}^{\circledR}{ }^{\circledR}$ Nacional Ossos, São Paulo, Brasil) e osso artificial de poliuretana Synbone ${ }^{\odot}$ (Synbone AG, Gotemburgo, Suécia). No estudo, foram utilizados diversos substratos para simular as diferentes densidades ósseas, deixando os resultados mais próximos da realidade clínica ${ }^{26-28}$. Além disso, a substituição dos ossos humanos permite uma maior padronização do estudo com o uso de poucas amostras, o que não ocorre com os ossos naturais, em razão de sua elevada variabilidade $^{6,29}$.

Para a confecção dos corpos de prova de osso suíno, foram utilizados 48 segmentos ósseos de $20 \mathrm{~mm}$ de comprimento, com aproximadamente a mesma espessura de osso cortical e esponjoso; foram, então, incluídos em cilindros de PVC (20 mm de diâmetro e $15 \mathrm{~mm}$ de altura) preenchidos com resina acrílica autopolimerizável, de forma que $5 \mathrm{~mm}$ do osso ficassem na parte externa do cilindro, não incluído pelo material. As amostras foram mantidas sob congelamento à temperatura de $-21 \pm 5{ }^{\circ} \mathrm{C}$ para manter suas propriedades físicas e, previamente aos procedimentos cirúrgicos e mensurações da estabilidade primária dos implantes, permaneceram em temperatura ambiente por duas horas, para atingir o equilíbrio térmico e não alterar suas propriedades biomecânicas. Embora alguns estudos adotem a metodologia de 48 horas de descongelamento para essa finalidade ${ }^{30}$, este trabalho seguiu orientações de Rosa et al. ${ }^{9}$ (2008), em função da larga utilização desse método na Ortopedia médica.

A madeira utilizada para a confecção dos corpos de prova foi o pinus, da espécie Auracária Augustifólia, selecionada pela sua anisotropia, qualidade presente no osso ${ }^{31}$, e por sua similaridade ao osso humano na perfuração e na instalação de implantes, devida, provavelmente, pela sua densidade básica ser de 0,42 a $0,48 \mathrm{~g} / \mathrm{cm}^{3}$, similar às médias dos ossos da mandíbula e da maxila ${ }^{32}$. Foram confeccionados corpos de prova cilíndricos com $13 \mathrm{~mm}$ de altura e $30 \mathrm{~mm}$ de diâmetro.

Os corpos de prova de poliuretano $\mathrm{Nacional}^{\circledR}$ nas três densidades (40, 20 e 15 PCF) foram obtidos em forma de blocos, com dimensões de $4,2 \mathrm{~cm}$ de espessura, $17,8 \mathrm{~cm}$ de largura e $6,5 \mathrm{~cm}$ de comprimento. As amostras da marca Synbone $e^{\odot}$ foram obtidas a partir do corte da cabeça do fêmur desse osso artificial, de forma que houvesse aproximadamente a mesma espessura de osso cortical e trabeculado, em todos os espécimes. 


\section{Seleção dos Implantes}

Foram utilizados 32 implantes da marca Conexão ${ }^{\circledR}$ (Conexão Sistemas de Prótese Ltda, Arujá, São Paulo, Brasil), divididos em quatro grupos $(n=8)$, conforme o formato, o tratamento de superfície e o tipo de conexão protética: Master Screw (MS), Master Porous (MP), Conect AR (CA) e Conect Cônico (CC) (Tabela 1).

Os implantes MS e CC apresentam superfície lisa ou usinada e os demais, MP e CA, possuem superfície tratada Porous, tratamento de superfície por meio de ataque ácido, o qual, segundo a literatura, aumenta a porcentagem de contato osso/ implante e o torque de inserção quando comparado aos implantes usinados $^{33,34}$. Os implantes Master Porous (MP) apresentam um tratamento de superfície denominado pela empresa como Porous $^{\circledR}$ duplo, mas a diferença entre este e o Porous ${ }^{\circledR}$ está relacionada apenas à marca comercial.

No estudo, os implantes cônicos analisados não possuíam tratamento de superfície em virtude da disponibilidade que a empresa oferece de implantes neste formato e diâmetro selecionado. Esse tipo de parafuso foi selecionado para que pudesse ser realizada comparação entre o formato cônico (CC) e o formato cilíndrico dos implantes (MS), sendo considerado o design o único fator de variação.

\section{Inserção dos Implantes}

Os implantes foram inseridos nos substratos por um único operador, seguindo-se a sequência de brocas cirúrgicas indicadas pelo fabricante. A perfuração foi iniciada a $7 \mathrm{~mm}$ da marcação do parafuso adjacente, de forma a evitar que a posição de um parafuso interferisse nas análises dos ensaios do outro.

\section{Quantificação da Estabilidade Primária}

As análises utilizadas para aferir a estabilidade primária foram o torque de inserção (TI), a resistência ao arrancamento (RA) e a frequência de ressonância (FR). Todos os grupos de implantes foram inseridos em todos os substratos, dos quais, após a análise do TI e da RA, foram removidos e inseridos em outro material. Os implantes MP e MS também foram analisados quanto à frequência de ressonância, sendo excluídos os outros grupos em razão da inexistência de um transdutor com adaptação às suas conformações de roscas.

As análises do TI foram feitas com o torquímetro digital $\operatorname{Kratos}^{\circledR}$ (Kratos Equipamentos Industriais Ltda, Cotia, SP, Brasil), acoplado aos dispositivos ou aos monta-implantes adaptados aos respectivos formatos dos parafusos. $\mathrm{O}$ valor foi mensurado a cada volta da inserção, sendo considerado o valor máximo obtido o maior TI. O número foi convertido em N.cm por meio da fórmula:

$$
\begin{aligned}
& y=0,0449 x-0.7907 \\
& \text { Em que: } \\
& y=o \text { valor em N.cm; } \\
& x=o \text { valor lido (rosa). }
\end{aligned}
$$

Esse método está baseado em uma metodologia empregada em teses já defendidas ${ }^{9}$, embora tenha sido planejado de acordo com a calibração do aparelho para análise em Oficina de Precisão.

A análise de $\mathrm{RA}^{21}$ usou os mesmos dispositivos e montaimplantes acoplados aos parafusos, por meio de roscas presentes nas duas estruturas. O corpo de prova com o implante inserido foi posicionado na parte inferior de uma base fixa de aço com um orifício no centro. O monta-implante ou o acessório desenvolvido foi parafusado e, em seguida, conectado à base móvel da máquina por meio de um pino, sendo então unidos a uma peça que serve para a adaptação na célula de carga $(200 \mathrm{~kg})$ da Máquina Universal de Ensaios EMIC ${ }^{\circledR}$ modelo DL-10000N. Cada implante foi submetido à RA por meio de força axial de tração com velocidade constante de $2 \mathrm{~mm} / \mathrm{min}$. Utilizando-se o programa Software Tesc 1.13, foi realizada a análise dos resultados por meio da curva 'força $\times$ deslocamento', obtida durante a realização dos testes mecânicos.

$\mathrm{Na}$ análise de FR, foram utilizados os implantes MP e MS. Foi avaliado o coeficiente de estabilidade (ISQ - Implant Stability Quotient) pela utilização do transdutor fixado sobre a plataforma do implante, por meio de um parafuso integrado. Através da excitação por impulsos magnéticos emitidos pela sonda de medição do instrumento, o aparelho emite um sinal sonoro e exibe o ISQ, sendo que o maior valor sugere uma maior estabilidade do implante. Foram feitas quatro medições ao redor de cada implante, obtendo-se uma média dos valores ${ }^{35-37}$.

Os dados foram analisados no que diz respeito à sua distribuição e à homogeneidade, e uma vez que eles foram considerados normais (teste de Kolmogorov-Smirnov) e homógenos (teste de Levene), foi realizada a análise estatística paramétrica com Teste ANOVA e Teste de Tukey (significância de 5\%), para a diferenciação entre as médias, a fim de se fazer a correlação entre o tratamento de superfície e o formato dos implantes, inseridos em diferentes substratos, através da associação de métodos, como TI, RA e FR.

Tabela 1. Grupos de implantes divididos de acordo com o formato, o tratamento de superfície e a conexão protética

\begin{tabular}{llccc}
\hline & Formato & Tratamento de Superfície & Conexão Protética & Tamanho \\
\hline Master Screw (MS) & Cilíndrico & Usinado & Hexágono Externo & $11,5 \times 3,75 \mathrm{~mm}$ \\
Master Porous (MP) & Cilíndrico & Porous ${ }^{\circledR}$ duplo & Hexágono Externo & $11,5 \times 3,75 \mathrm{~mm}$ \\
Conect AR (CA) & Cilíndrico & Porous ${ }^{\circledR}$ & Hexágono Interno & $11,5 \times 3,75 \mathrm{~mm}$ \\
Conect Cônico (CC) & Cônico & Usinado & Hexágono Externo & $11,5 \times 3,5 \mathrm{~mm}$ \\
\hline
\end{tabular}




\section{RESULTADO}

Ao analisar a influência do formato e do tratamento de superfície do implante no TI, foi observado que, quando inserido no osso de costela suína, CC apresentou maior média de torque de inserção (12,35 $\pm 2,74)$, seguido do CA $(12,14 \pm 1,03)$, com diferença estatística para o MP $(6,52 \pm 1,12)(\mathrm{p}<0,05)$. O osso artificial de poliuretana Synbone ${ }^{\odot}$ apresentou melhor desempenho para os implantes cilíndricos e menor média para o implante cônico, com TI $(4,45 \pm 0,82)$, sendo diferente estatisticamente de todos os cilíndricos. Na poliuretana $\mathrm{Nacional}^{\circledR}$, nas densidades de 40 PCF e 20 PCF, os implantes cilíndricos também tiveram maior média de TI e o implante cônico, a menor média de torque $(10,23 \pm 1,81)$. Na menor densidade, 15 PCF, apesar de CA apresentar a maior média de TI $(9,36 \pm 1,36)$, não houve diferença estatística com todos os outros grupos. Na madeira, os implantes de hexágono externo, cilíndricos e cônicos tiveram a maior média de torque. Comparando-se todos os implantes inseridos em todos os substratos, MP e MS tiveram a média mais elevada de TI na poliuretana Nacional, de 40 PCF. De maneira geral, os implantes cilíndricos apresentaram os maiores resultados de TI em qualquer substrato, com exceção do osso suíno, em que CC teve o maior TI, sem, contudo, apresentar diferença estatística com os implantes MS e CA. Além disso, MS e MP sempre se apresentaram iguais estatisticamente, independentemente da presença do tratamento de superfície (Figura 1).

Os resultados da RA dos implantes inseridos no osso suíno e na poliuretana Synbone ${ }^{\odot}$ mostraram que não houve diferença estatística entre todos os tipos de implantes. No substrato de poliuretana $\mathrm{Nacional}^{\circledR}$ de $40 \mathrm{PCF}$, CA teve maior média $(1.463,21 \pm 122,84 \mathrm{~N})$ e foi diferente estatisticamente de todos os demais $(\mathrm{p}<0,05)$. O implante cônico teve resultado de RA inferior aos outros $(491,68 \pm 75,91 \mathrm{~N})$, entretanto, foi igual estatisticamente a $\operatorname{MP}(603,10 \pm 190,33 \mathrm{~N})$ e MS $(612,73 \pm 49,22 \mathrm{~N})$. Na densidade de 20 PCF, CA também teve o maior resultado $(347,10 \pm 22,34 \mathrm{~N})$, no entanto, foi diferente estatisticamente de $\mathrm{MP}(61,77 \pm 20,35 \mathrm{~N})$ $(\mathrm{p}=0,00)$ e MS $(45,52 \pm 18,79 \mathrm{~N})(\mathrm{p}=0,00)$. CC, que não apresentou a menor média de força de arrancamento $(206,57 \pm 31,51 \mathrm{~N})$, não teve diferença estatística com os implantes cilíndricos. A maior média de RA foi observada no CA, quando inserido na poliuretana Nacional ${ }^{\circledR}$ de $15 \operatorname{PCF}(227,79 \pm 33,92 \mathrm{~N})$, seguido de

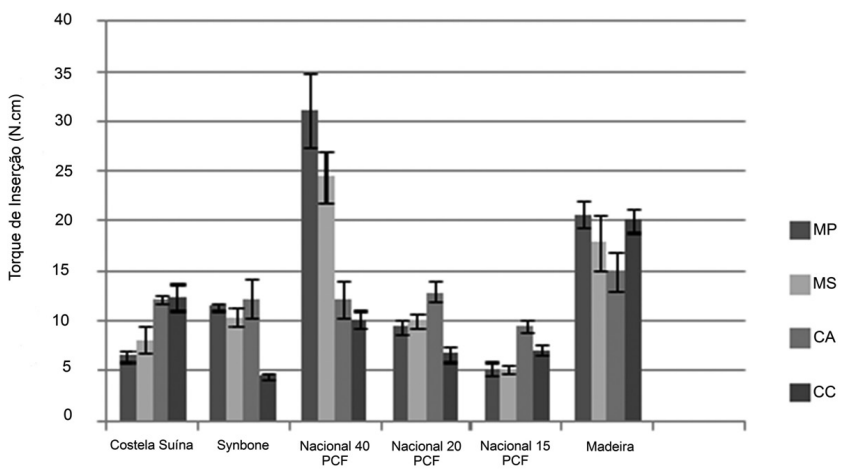

Figura 1. Gráfico ilustrando o torque de inserção dos implantes (N.cm)
$\mathrm{CC}(209,57 \pm 9,60 \mathrm{~N})(\mathrm{p}>0,05)$. MS, que mostrou menor média $(33,13 \pm 19,94)$, teve diferença estatística com estes implantes de maior média ( $\mathrm{p}=0,01$ para $\mathrm{CA}$ e $\mathrm{p}=0,04$ para $\mathrm{CC}$ ), assim como MP também teve e apresentou diferença com MS $(p<0,05)$. Na madeira, implantes cilíndricos com tratamento de superfície apresentaram maior média de RA e CC teve a menor média $(483,00 \pm 82,32 \mathrm{~N})$, sendo diferente estatisticamente dos de maior média $(\mathrm{p}=0,00)$. De modo geral, com exceção do osso, CA teve a maior média de RA em todos os substratos, sendo que, no osso Synbone $^{\odot}$ e Nacional $^{\circledR}$ de 20 e 15 PCF, CC não tinha diferença significante com este (Figura 2).

Após análise estatística dos valores obtidos na FR, foi constatado que, apesar de MP apresentar maior média de ISQ que MS em todos os substratos, estes não apresentaram diferença estatística entre si, com exceção de quando inseridos na poliuretana Nacional de 20 PCF ( $\mathrm{p}=0,01)$ (Figura 3).

\section{DISCUSSÃO}

A interação entre o implante e o osso adjacente imediatamente após sua inserção depende, principalmente, do formato do parafuso e da composição química da sua superfície ${ }^{32,38}$. Embora a biocompatibilidade do titânio seja uma condição prévia para o sucesso clínico da terapia com implantes, é a propriedade osteocontudora a responsável por acelerar a osseointegração e

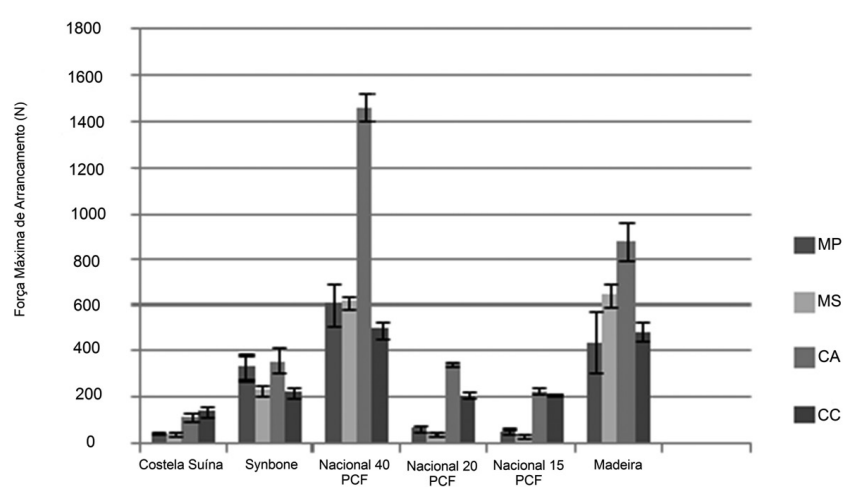

Figura 2. Gráfico ilustrando a força máxima de arrancamento $(\mathrm{N})$ dos implantes

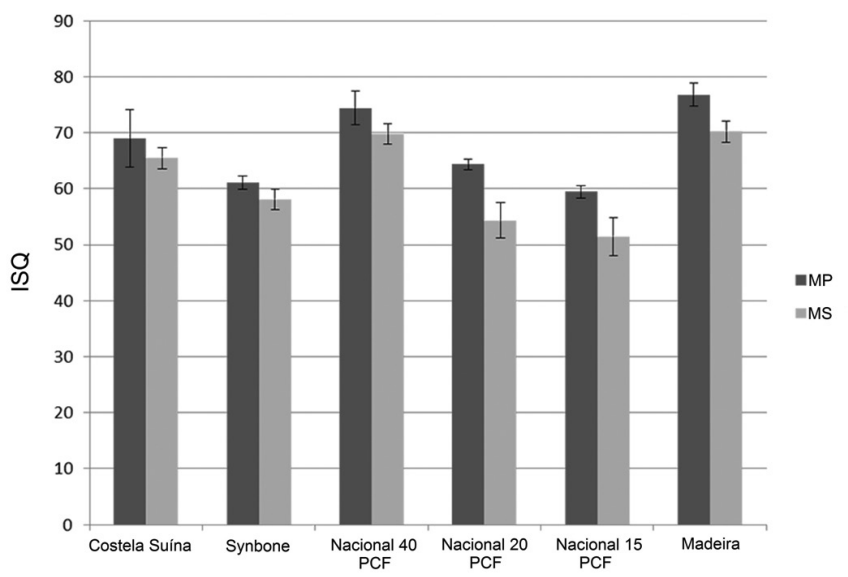

Figura 3. Gráfico ilustrando o quociente de estabilidade primária (ISQ) dos implantes 
melhorar a estabilidade biomecânica. Uma das formas de elevar a osteocondutividade do implante é aumentar a rugosidade superficial, por meio de jateamento de partículas, ataque ácido, anodização da superfície e até pela alteração morfológica do implante ${ }^{39}$.

Por outro lado, alguns estudos na literatura ainda questionam a influência do tratamento de superfície na estabilidade primária ${ }^{40-43}$, corroborando com os resultados do presente estudo, para os quais, quando comparados com os implantes MP (com superfície tratada) e os MS (sem superfície tratada), não foi verificada diferença estatisticamente significante entre os tipos testados quanto ao TI, o que está de acordo com achados da literatura ${ }^{43}$, ao verificarem que a estabilidade primária foi maior para implantes usinados ${ }^{40,44}$, provavelmente pela condição in vitro da análise.

Diversos autores ${ }^{45,46}$ avaliam a força e a rigidez do cisalhamento na interface osso-implante, através da RA, em busca de informações a respeito do grau de contato nessa interface ${ }^{40}$. No presente estudo, ao avaliar a RA dos implantes inseridos na poliuretana Nacional de $40 \mathrm{PCF}$, verificou-se que os implantes CA obtiveram os maiores valores, sugerindo um melhor desempenho em substratos de maior densidade. Esse resultado, provavelmente, se deve à maior superfície de contato e atrito entre o parafuso e o material ${ }^{40,47}$. Apesar do melhor desempenho em RA do implante com tratamento de superfície, na poliuretana Nacional ${ }^{\circledR}$ de $40 \mathrm{PCF}$, não houve diferença estatística entre os implantes de mesmo formato e sem tratamento de superfície (MS). Portanto, sugere-se que os implantes CA apresentaram maior média em função do seu formato ou, mais provavelmente, do tipo de conexão protética. Os resultados nulos da influência do tratamento de superfície na RA têm como hipótese o fato de a magnitude da força aplicada ser tão elevada que a rugosidade ou o aumento da espessura da camada superficial dos implantes não seria capaz de resistir significantemente à aplicação dessa força ${ }^{23,48}$.

$\mathrm{Na}$ análise FR, foram observados valores maiores em implantes com tratamento em comparação aos usinados ${ }^{37}$, que mostraram relação dos valores de ISQ com a espessura de óxidos e o tamanho do microporo. Apesar desses dados, a presença do tratamento de superfície não apresentou diferença significativa entre os implantes usinados e tratados em todos os substratos, com exceção da poliuretana de 20 PCF. Esses resultados corroboram com estudos presentes na literatura ${ }^{8,40}$ e sugerem como hipótese o fato de a FR não ter sensibilidade suficiente para detectar alterações menores, como o tratamento de superfície de implantes.

Alguns estudos demonstram que, em função da compressão lateral na cortical de sítios ósseos com baixa qualidade, implantes cônicos têm um maior TI do que cilíndricos ${ }^{14,19,11-49}$. Essa afirmação condiz com os resultados encontrados no presente estudo, em que os implantes cônicos apresentaram maior TI, comparados aos cilíndricos, quando inseridos em osso suíno e em ossos artificiais de poliuretano de 15 PCF, sugerindo a utilização desse tipo de parafuso em osso de baixa densidade ${ }^{11}$. No estudo, foram utilizados apenas implantes cônicos sem tratamento de superfície, pois a empresa não oferecia disponibilidade para o tratamento nesse formato e diâmetro selecionado.

$\mathrm{Na}$ madeira, a maior média de torque foi encontrada em implantes cilíndricos e, quando comparados entre si, verificouse que os implantes do tipo hexágono interno apresentaram os menores valores. Embora a plataforma protética não influencie na estabilidade primária ${ }^{35}$, a diferença no formato da mesma exige que a geometria externa do parafuso (profundidade, número e passo de rosca) seja também diferente, para garantir as propriedades físico-químicas e mecânicas, e a resistência necessária. Dessa forma, a conformação das espiras parece ter influenciado mais do que o próprio formato do parafuso no torque de inserção ${ }^{8,14}$. Assim, justifica-se a utilização, no presente estudo, de implantes de mesmo formato e tratamento de superfície, porém com plataformas protéticas diferentes, como os implantes MP e CA.

Possivelmente, em função da utilização de diferentes substratos para a inserção dos implantes, os resultados da estabilidade primária foram diferentes dos encontrados comumente na literatura, segundo a qual os implantes cônicos apresentam maior torque de inserção, quando comparados aos cilíndricos ${ }^{4,14,19,11,49}$.

Assim como no TI, ao avaliar a RA de implantes ortopédicos, alguns estudos apresentam maiores valores em parafusos cônicos que cilíndricos ${ }^{23}$. No presente estudo, essa característica só foi observada quando o dispositivo utilizado para o ensaio era diferente. Além disso, deve-se ressaltar o uso do implante cônico com diâmetro menor neste estudo, por causa da disponibilidade da empresa, o que pode ter influenciado nos menores valores de TI e RA, pois, segundo alguns autores ${ }^{23}$, parafusos com o desenho cônico podem apresentar propriedades mecânicas inferiores aos parafusos cilíndricos, quando comparados em diferentes dimensões.

Além disso, o presente estudo avaliou somente os aspectos mecânicos do efeito do formato e do tratamento de superfície e, com isso, não considerou os fatores biológicos, como a resposta óssea, as características individuais, as variações locais do osso humano e a técnica cirúrgica, variáveis essas que também influenciam na estabilidade primária em uma situação clínica. Confirma-se, assim, que a melhor forma de usufruir dos benefícios oferecidos pelos diversos tratamentos de superfície, formatos e diferenças nas roscas dos parafusos depende da correlação entre formato e densidade óssea, para a promoção de uma condição biomecânica ótima para a osseointegração.

\section{CONCLUSÃO}

Os resultados obtidos, com a metodologia aplicada, permitiram concluir que os formatos e o tratamento de superfície estudados não demonstraram valores significantes, quando comparados os implantes entre si. Conclui-se também que, para os substratos avaliados, não houve diferença estatística entre os diferentes tipos de implantes testados. 


\section{REFERÊNCIAS}

1. Aksoy U, Eratalay K, Tözüm TF. The possible association among bone density values, resonance frequency measurements, tactile sense, and histomorphometric evaluations of dental implant osteotomy sites: a preliminary study. Impl Dent. 2009; 18(4): 316-25. PMid:19667820. http://dx.doi.org/10.1097/ID.0b013e31819ecc12

2. Brouwers JEIG, Lobbezoo F, Visscher CM, Wismeijer D, Naeije M. Reliability and validity of the instrumental assessment of implant stability in dry human mandibles. J Oral Rehabil. 2009; 36(4): 279-83. PMid:19220717. http://dx.doi.org/10.1111/j.1365-2842.2009.01944.x

3. Camacho FMT, Sakakura CE, Mera MFM, Esteves JC, Ribeiro FS, Pontes AEF. Avaliação clínica em curto prazo do sistema de Cone Morse e plataforma reduzida em prótese sobre implantes do tipo protocolo: estudo piloto randomizado controlado. Rev Odontol UNESP. 2012; 41(4): 247-53.

4. Chong L, Khocht A, Suzuki JB, Gaughan J. Effect of implant design on initial stability of tapered implants. J Oral Implantol.2009; 35(3): 130-5. PMid:19579524. http://dx.doi.org/10.1563/1548-1336-35.3.130

5. Degidi M, Giuseppe D, Adriano P. Determination of primary stability: a comparison of the surgeon's perception and objective measurements. Int J Oral Maxillofac Implants. 2010; 25(3): 558-61. PMid:20556255.

6. Mazzo CR, Reis AC, Shimano AC, Valente ML. In vitro analysis of the influence of surface treatment of dental implants on primary stability. Braz Oral Res. 2012; 26(4): 313-17. http://dx.doi.org/10.1590/S1806-83242012005000006

7. Morton D, Jaffin R, Weber H-P. Immediate restoration and loading of dental implants: clinical considerations and protocols. Int J Oral Maxillofac Implants. 2004; 19: 103-8. PMid:15635950.

8. Santos MV, Elias CN, Cavalcanti Lima JH. The effects of superficial roughness and design on the primary stability of dental implants. Clin Implant Dent Relat Res. 2011; 13(3): 215-23. PMid:19744197. http://dx.doi.org/10.1111/j.1708-8208.2009.00202.x

9. Rosa RC, Silva P, Shimano AC, Volpon JB, Defino HLA, Schleicher P, et al. Biomechanical analysis of the variables related to the pullout strength of screws in the vertebral fixation system. Rev Bras Ortop. 2008; 43: 293-9. http://dx.doi.org/10.1590/S0102-36162008000700005

10. Desai SR, Desai MS, Katti G, Karthikeyan I. Evaluation of design parameters of eight dental implant designs: a two-dimensional finite element analysis Níger. J Clin Pract. 2012; 15(2): 176-81.

11. Aleo E, Varvara G, Scarano A, Sinjari B, Murmura G. Comparison of the primary stabilities of conical and cylindrical endosseous dental implants: an in-vitro study. J Biol Regul Homeost Agents. 2012; 26(1): 89-96. PMid:22475100.

12. Tetè S, Zizzari V, De Carlo A, Sinjari B, Gherlone E. Macroscopic and microscopic evaluation of a new implant design supporting immediately loaded full arch rehabilitation. Ann Stomatol (Roma). 2012; 3(2): 44-50.

13. Carvalho BM, Pellizzer EP, Moraes SLD, Falcón-Antenucci RM, Ferreira JS Jr. Surface treatments in dental implants. Rev Cir Traumat Buco-Maxilo-Fac. 2009; 9(1): 123-30.

14. Bezerra F, Ribeiro EDP, Sousa SB, Lenharo A. Influence of macro-geometry in the primary stability of implants. Innov Implant J Biomater Esthet. 2010; 15(1): 29-34.

15. Wennerberg A, Albrektsson T, Johansson C, Andersson B. Experimental study of turned and grit-blasted screw-shaped implants with special emphasis on effects of blasting material and surface topography. Biomaterials. 1996; 17(1): 15-22. http://dx.doi.org/10.1016/01429612(96)80750-2

16. Tabassum A, Meijer GJ, Wolke JGC, Jansen JA. Influence of surgical technique and surface roughness on the primary stability of an implant in artificial bone withdifferent cortical thickness: a laboratory study. Clin Oral Implants Res. 2010; 21(2): 213-220. PMid:20070754. http:// dx.doi.org/10.1111/j.1600-0501.2009.01823.x

17. Ahmad OK, Kelly JR. Assessment of the primary stability of dental implants in artificial bone using resonance frequency and percussion analyses. Int J Oral Maxillofac Implants. 2013; 28(1): 89-95. PMid:23377052. http://dx.doi.org/10.11607/jomi.2554

18. Gabay E, Cohen O, Machtei EE. A novel device for resonance frequency assessment of one-piece implants. Int J Oral Maxillofac Implants. 2012; 27(3): 523-27. PMid:22616044.

19. Kahraman S, Bal BT, Asar NV, Turkyilmaz I, Tözüm TF. Clinical study on the insertion torque and wireless resonance frequency analysis in the assessment of torque capacity and stability of self-tapping dental implants. J Oral Rehabil. 2009; 36(10): 755-61. PMid:19758410. http://dx.doi.org/10.1111/j.1365-2842.2009.01990.x

20. Turkyilmaz I, Mcglumphy EA. Influence of bone density on implant stability parameters and implant success: a retrospective clinical study. BMC Oral Health. 2008 24; 8: 32.

21. American Society for Testing Materials. Standard Specification and Test Methods for Metallic Medical Bone Screws. Report: F543 [cited 2011 Jan 20]. Available from: http://www.astm.org

22. Salmória KK, Tanaka OM, Guariza-Filho O, Camargo ES, de Souza, LT, Maruo, H. Insertional torque and axial pull-out strength of miniimplants in mandibles of dogs. Am J Orthod Dentofacial Orthop. 2008; 133(6): 790.e15-22.

23. Zamarioli A, Simões PA, Shimano AC, Defino HLA. Insertion torque and pullout strength of vertebral screws with cylindrical and conic core. Rev Bras Ortop. 2008; 43(10): 452-59. http://dx.doi.org/10.1590/S0102-36162008001000005

24. Cristofolini L, Viceconti M. Mechanical validation of whole composite tibia models. J Biomechanics. 2000; 33(3): 279-88. http://dx.doi. org/10.1016/S0021-9290(99)00186-4 
25. Oliscovicz NF, Shimano AC, Marcantonio Junior E, Lepri CP, Reis AC. Analysis of primary stability of dental implants inserted in different substrates using the pullout test and insertion torque. Int J Dent (Online). 2013 (2013), Article ID 194987, 5 pages http://dx.doi. $\operatorname{org} / 10.1155 / 2013 / 194987$

26. Marquezan M, Osório A, Sant’anna E, Souza MM, Maia L. Does boné mineral density influence the primary stability of dental implants? A systematic review. Clin Oral Implants Res. 2012; 23(7): 767-74.

27. Isoda K, Ayukawa Y, Tsukiyama Y, Sogo M, Matsushita Y, Koyano K. Relationship between the bone density estimated by cone-beam computed tomography and primary stability of dental implants. Clin Oral Implants Res. 2012; 23(7): 832-36. PMid:21635560. http:// dx.doi.org/10.1111/j.1600-0501.2011.02228.x

28. Turkyilmaz I, Company AM. Sensitivity of resonance frequency analysis method to assess implant stability. N Y State Dent J. 2011; 77(5): 44-9. PMid:21545533. http://dx.doi.org/10.1111/j.1600-0501.2011.02203.x

29. Vasconcellos LG, Nishioka RS, Vasconcellos LM, Nishioka LN. Effect of axial loads on implant-supported partial fixed prostheses by strain gauge analysis. J Appl Oral Sci. 2011; 19(6): 610-15. PMid:22230995.

30. Akkocaoglu M, Uysal S, Tekdemir I, Akca K, Cehreli MC. Implant design and intraosseous stability of immediately placed implants: a human cadaver study. Clin Oral Implants Res. 2005; 16(2): 202-9. PMid:15777330. http://dx.doi.org/10.1111/j.1600-0501.2004.01099.x

31. Pfeil W, Pfeil M. Estruturas de madeira. 6a ed. Rio de Janeiro: Ed. LTC; 2003.

32. Tabassum A, Meijer GJ, Wolke JGC, Jansen JA. Influence of surgical technique and surface roughness on the primary stability of an implant in artificial bone with different cortical thickness: a laboratory study. Clin Oral Implants Res. 2010; 21(2): 213-20. PMid:20070754. http:// dx.doi.org/10.1111/j.1600-0501.2009.01823.x

33. Grassi S, Piattelli A, Figueiredo LC, Feres M, de Mello L, Iezzi G, et al. Histologic evaluation of earlybone response to different implant surfaces. J Periodontol. 2006; 77(10): 1736-43. PMid:17032118. http://dx.doi.org/10.1902/jop.2006.050325

34. Martínez González JM, García Sabán F, Ferrándiz Bernal J, Gonzalo Lafuente JC, Cano Sánchez J, Barona Dorado C. Removal torque and physico-chemical characteristics of dental implants etched with hydrofluoric and nitric acid. An experimental study in Beagle dogs. Med Oral Patol Oral Cir Bucal. 2006; 11(3): E281-5. PMid:16648769.

35. Cehreli MC, Karasoy D, Akca K, Eckert SE. Meta-analysis of methods used to assess implant stability. Int J Oral Maxillofac Implants. 2009; 24(6): 1015-32. PMid:20162105.

36. Kahraman S, Bal BT, Asar NV, Turkyilmaz I, Tözüm TF. Clinical study on the insertion torque and wireless resonance frequency analysis in the assessment of torque capacity and stability of self-tapping dental implants. J Oral Rehabil. 2009; 36(10): 755-61. PMid:19758410. http://dx.doi.org/10.1111/j.1365-2842.2009.01990.x

37. Rozé J, Babu S, Saffarzadeh A, Gayet-Delacroix M, Hoornaert A, Layrolle P. Correlating implant stability to bone structure. Clin Oral Implants Res. 2009; 20(10): 1140-5. PMid:19519789. http://dx.doi.org/10.1111/j.1600-0501.2009.01745.x

38. Barros RRM, Novaes Jr AB, Papalexiou V, Souza SLS, Taba M Jr., Palioto DB, et al. Effect of biofunctionalized implant surface on osseointegration - a histomorphometric study in dogs. Braz Dent J. 2009; 20(2): 91-8. http://dx.doi.org/10.1590/S0103-64402009000200001

39. Hofstetter W, Sehr H, Wild MD, Portenier J, Gobrecht J, Hunziker EB. Modulation of human osteoblasts by metal surface chemistry. J Biomed Mater Res A. 2013 Aug;101(8):2355-64. doi: 10.1002/jbm.a.34541. Epub 2013 Jan 28. http://dx.doi.org/10.1002/jbm.a.34541

40. Kim S-J, Kim M-R, Rim J-S, Chung S-M, Shin SW. Comparison of implant stability after different implant surface treatments in dog bone. J Appl Oral Sci. 2010; 18(4): 415-20. http://dx.doi.org/10.1590/S1678-77572010000400016

41. Cooper LF. Factors influencing primary dental implant stability remain unclear. J Evid Based Dent Pract. 2012; 12(3 Suppl): 185-6. http:// dx.doi.org/10.1016/S1532-3382(12)70033-7

42. Prodanov L, Lamers E, Wolke J, Huiberts R, Jansen JA, Frank Walboomers X. In vivo comparison between laser-treated and grit blasted/ acid etched titanium. Clin Oral Implants Res. 2013 Jan 25. doi: 10.1111/clr.12109. [Epub ahead of print] http://dx.doi.org/10.1111/ clr.12109

43. Da Cunha H, Francischone CE, Nary H Filho, Oliveira RCG. A comparison between insertion torque and resonance frequency in the assessment of primary stability and final torque capacity of standard and TiUnite single-tooth implants under immediate loading. Int $\mathrm{J}$ Oral Maxillofac Implants. 2004; 19(4): 578-85. PMid:15346756.

44. Tabassum A, Meijer GJ, Wolke JGC, Jansen JA. Influence of the surgical technique and surface roughness on the primary stability of an implant in artificial bone with a density equivalent to maxillary bone: a laboratory study. Clin Oral Implants Res. 2009; 20(4): 327-32. PMid:19298286. http://dx.doi.org/10.1111/j.1600-0501.2008.01692.x

45. Elmengaard B, Bechtold JE, Soballe KS. In vivo study of the effect of treatment on bone on growth on press-fit titanium alloy implants. Biomaterials. 2005; 26(17): 3521-6. PMid:15621242. http://dx.doi.org/10.1016/j.biomaterials.2004.09.039

46. Rabel A, Köhler SG, Schmidt-Westhausen AM. Clinical study on the primary stability of two dental implant systems with resonance frequency analysis. Clin Oral Investigations. 2007; 11(3): 257-65. PMid:17401588. http://dx.doi.org/10.1007/s00784-007-0115-2

47. Gedrange T, Hietschold V, Mai R, Wolf P, Nicklish M, Harzer W. An evaluation of resonance frequency analysis for the determination of the primary stability of orthodontic palatal implants. A study in human cadavers. Clin Oral Implants Res. 2005; 16(4): 425-31. PMid:16117766. http://dx.doi.org/10.1111/j.1600-0501.2005.01134.x

48. Leite VC, Shimano AC, Gonçalves GAP, Kandziora F, Defino HLA. The influence of insertion torque on pedicular screws' pullout resistance. Acta Ortop Bras. 2008; 16: 214-6. http://dx.doi.org/10.1590/S1413-78522008000400005

49. Sakoh J, Wahlmann U, Stender E, Al-Nawas B, Wagner W. Primary stability of a conical implant and a hybrid,cylindric screw-type implant in vitro. Int J Oral Maxillofac Implants. 2006; 21(4): 560-6. PMid:16955606. 


\section{CONFLITOS DE INTERESSE}

Os autores declaram não haver conflitos de interesse.

\section{AUTOR PARA CORRESPONDÊNCIA}

Andrea Candido dos Reis

Departamento de Materiais Dentários e Prótese, Faculdade de Odontologia, USP - Universidade de São Paulo, Av. do Café, s/n, 14040-904 Ribeirão Preto-SP, Brasil

e-mail: andreare73@yahoo.com.br

Recebido: 04/03/2013

Aprovado: 25/07/2013 